\title{
Obstrução de vias lacrimais associada ao tratamento radioiodoterápico de carcinoma de tireoide
}

\author{
Lacrimal drainage system obstruction associated to radioactive iodine therapy for thyroid carcinoma
}

Fabricio Lopes da Fonseca ${ }^{1}$, Patrícia Lunardelli ${ }^{1}$, Suzana Matayoshi ${ }^{1}$

\section{RESUMO}

Objetivo: Descrever uma série de pacientes portadores de obstrução do sistema lacrimal associado à radioiodoterapia para tratamento de carcinoma de tireoide, revisar os dados clínicos e a resposta ao tratamento cirúrgico desta rara complicação. Métodos: Foi realizada uma análise retrospectiva dos achados oftalmológicos de pacientes com histórico de carcinoma de tireoide previamente submetidos à tireoidectomia e à RIT que foram encaminhados para cirurgia de vias lacrimais.

Resultados: Dezessete pacientes com carcinoma de tireoide tratados com tireoidectomia e RIT apresentaram obstrução do ducto nasolacrimal sintomática após período médio de 13,2 meses do tratamento do câncer. Onze pacientes tiveram epífora bilateral, 8 com mucocele de saco lacrimal. A idade dos pacientes variou entre 30 e 80 anos, sendo 10 com idade menor ou igual a 49 anos. A dose cumulativa média de radioiodo administrada foi de 571 mCi (variação entre 200-1200 mCi). Sintomas de obstrução nasal e aumento de glândulas salivares ocorreram em 53\% dos pacientes. Todos os pacientes foram submetidos à dacriocistorrinostomia. Observou-se ainda que nos 3 pacientes mais jovens houve maior sangramento intraoperatótio e dilatação de saco lacrimal. A resolução completa da epífora e da dacriocistite ocorreu em 82,4\%, e foi parcial em 17,6\% (3 pacientes mantiveram queixa unilateral após a correção da obstrução bilateralmente). O seguimento médio foi de 6 meses (intervalo: 2-24 meses).

Conclusões: Alta dose cumulativa de radioiodo, disfunção nasal e de glândulas salivares estão associadas à obstrução das vias lacrimais. Observa-se uma maior porcentagem de pacientes mais jovens apresentando quadro de dacriocistite quando comparado à dacrioestenose idiopática. Aabsorção de iodo radioativo pela mucosa do ducto nasolacrimal com subsequente inflamação, edema e fibrose parece ter relação direta com a obstrução do ducto nasolacrimal. O conhecimento desta complicação é importante para o estudo e abordagem correta desses pacientes.

Descritores: Ducto nasolacrimal/efeitos de radiação; Doenças do aparelho lacrimal; Neoplasias da glândula tireoide/radioterapia; Radioisótopos de iodo

\section{ABSTRACT}

Purpose: To report the finding of nasolacrimal drainage system obstruction associated with radio iodine therapy and to review clinical data and the surgical treatment outcome of this rare complication.

Methods: We retrospectively analyzed ophthalmological data of patients with history of thyroid carcinoma that underwent radioactive iodine 1-131 therapy and were referred to lacrimal surgery.

Results: 17 patients with thyroid cancertreated with thyroidectomy and radioactiveiodine 1-131 therapy presented symptomatic nasolacrimal duct obstruction after 13.2 months following cancer treatment. 11 patients presented bilateral epiphora, 8 had lacrimal sac mucocele. Age range was 30 to 80 years, 10 patients had less than or equal to 49 years. The mean cumulative dose of radioiodine was $571 \mathrm{mCi}$ (range: 200-1200 mCi). Nasal obstruction symptoms and increased salivary glands were also present in $53 \%$ of patients. All subjects underwent dacryocystorhinostomy. Dilation of the lacrimal sac and increased intraoperative bleeding was also observed in 3 younger patients. Complete epiphora and dacryocystitis resolution after surgery occurred in $82.4 \%$, and partial in 17.6\% (3 patients that still presented unilateral relapse after correction of bilateral obstruction). Mean follow-up was 6 months (range: 2-24 months).

Conclusions: Cumulative high dose ofradioidine, nasal and salivarygland dysfunction are associated with lacrimal drainage obstruction. We observed a great percentage of younger patients presenting dacryocystitis when compared to the idiopathic dacryostenosis. Radioactive iodine uptake by nasolacrimal duct mucosa with subsequent inflammation, edemaandfibrosis seems to havearelationship tolacrimalductobstruction. The knowledge of this complication is important for the study and proper management of these patients.

Keywords: Nasolacrimal duct/radiation effects; Lacrimal apparatus diseases; Thyroid neoplasms/radiotherapy; lodine radioisotopes

\section{INTRODUÇÃO}

A obstrução de pontos lacrimais e canalículos é relatada como complicação de tratamentos oncológicos com diversas drogas, dentre as quais docetaxel, citosina arabinosídeo, 5-fluoruracil, metotrexate e mitomicina- $C^{(1)}$.

O uso da radioiodoterapia (RIT) com iodo 131, juntamente com o tratamento cirúrgico (tireoidectomia), constituem a abordagem corrente de carcinomas diferenciados de tireoide. O câncer de tireoide corresponde a 1,5\% das neoplasias malignas, atingindo entre 8.000 e 14.000 novos casos ao ano nos Estados Unidos da América ${ }^{(2)}$. A doença atinge mais frequentemente mulheres (3F:1M), acima de 45 anos de idade, sendo o carcinoma papilífero e o carcinoma folicular os tipos histológicos mais comuns (75\% dos casos)(2). É importante salientar o aumento de incidência em todo mundo, sendo que nos EUA observou-se que nos últimos 30 anos houve um aumento de 2,4 vezes, passando de 3,6 para 8,7 casos por 100.000 habitantes $^{(3)}$.

As complicações sistêmicas da RIT geralmente são leves e fugazes, sendo bem estabelecido o acometimento de glândulas salivares com subsequente xerostomia ${ }^{(4,5)}$. As complicações oculares são pouco relatadas ${ }^{(6)}$, sendo descritas conjuntivite crônica e recorrente, ceratoconjuntivite seca e xeroftalmia ${ }^{(7)}$. A disfunção de glândula lacrimal é descrita em estudos recentes, notadamente após alta dose cumulativa do fármaco ${ }^{(8,9)}$. Entretanto, a utilização de radiofármacos não era apresentada como fator causal, havendo poucos estudos que

Submetido para publicação: 15 de setembro de 2011

Aceito para publicação: 12 de janeiro de 2012

Trabalho realizado no Setor de Plástica Ocular, Divisão de Clínica Oftalmológica, Hospital das Clínicas, Faculdade de Medicina,Universidade de São Paulo - USP, São Paulo (SP), Brasil.

Médico, Setor de Plástica Ocular, Divisão de Clínica Oftalmológica, Hospital das Clínicas, Faculdade de Medicina,Universidade de São Paulo - USP - São Paulo (SP), Brasil.

Financiamento: Não houve fianciamento para este estudo.

Divulgação de potenciais conflitos de interesse: F.L.Fonseca, Não; P.Lunardelli, Não, S.Matayoshi, Não.

Endereço de correspondência: Suzana Matayoshi. Rua Harmonia, 1177 - São Paulo (SP) - 05435001 - Brazil - E-mail: suzana.matayoshi@gmail.com 
abordem a sua relação com obstrução de vias lacrimais, notando-se especial ausência do tema na literatura nacional.

O objetivo desse estudo é apresentar uma série de casos de obstrução lacrimal em pacientes portadores de carcinoma de tireoide tratados com tireoidectomia e radioiodoterapia, descrever as características clínicas e a evolução desses pacientes.

\section{MÉTODOS}

Apresentamos uma série retrospectiva de 17 indivíduos com diagnóstico de carcinoma diferenciado de tireoide e submetidos a tireoidectomia e RIT. A amostra foi obtida pelo levantamento de prontuários de pacientes encaminhados com diagnóstico de obstrução lacrimal sintomática no período de janeiro de 2008 a janeiro de 2010 para o ambulatório dos autores. A avaliação lacrimal constou de biomicroscopia do filme lacrimal, sondagem e irrigação lacrimal, complementadas por dacriocistografia e nasofibroscopia. Foram coIhidos dados referentes à epífora, secreção em saco lacrimal, xerostomia, parotidite, ressecamento nasal e aumento de glândulas salivares. A presença de metástases e a dose cumulativa de radioiodo foram registrados. O intervalo entre a última sessão de radioiodoterapia e a instalação de epífora/secreção também foram avaliados.

A obstrução lacrimal foi classificada quanto à topografia (alta ou baixa), às dimensões do saco lacrimal (graduação de 1 a 4 conforme dimensões observadas à dacriocistografia) e à lateralidade (uni ou bilateral). As complicações intraoperatórias, bem como recidivas, foram analisadas durante o período de seguimento.

\section{RESULTADOS}

Dezessete prontuários de pacientes foram analisados, sendo todos casos de obstrução lacrimal em pacientes com histórico de carcinoma de tireoide submetidos a tratamento cirúrgico e RIT nos últi- mos 3 anos. Esses prontuários foram selecionados a partir de análise de 74 prontuários de pacientes submetidos à dacriocistorrinostomia pelos autores no período. A idade variou entre 30 e 80 anos, com 59\% dos pacientes (10 indivíduos) com idade menor ou igual a 49 anos (Tabela 1). Quanto ao gênero, 82,3\% dos pacientes (14 indivíduos) eram do sexo feminino. Todos os pacientes apresentaram queixa espontânea de epífora, motivo pelo qual foram encaminhados para avaliação. Doze pacientes apresentavam secreção à expressão da região do saco lacrimal com sinais de dacriocistite crônica.

A dose cumulativa de radioiodomédia foi de $571 \mathrm{mCi}$, variando entre 200 e 1200 mCi, sendo que dose maior e maior número de sessões ocorreram nos casos onde havia metástases (cinco pacientes: caso 5 com dose cumulativa de 960 mCi e metástase pulmonar; caso 6 com dose de $1200 \mathrm{mCi}$ e acometimento de linfonodo cervical; caso $10 \mathrm{com}$ dose de $670 \mathrm{mCi}$ e metástase em mediastino; caso $13 \mathrm{com}$ dose de 675 mCi e metástase pulmonar, hepática e linfonodal, e o caso 8 com dose de $650 \mathrm{mCi}$ e acometimento de linfonodo cervical).

O intervalo médio entre a última dose de radioiodo e a instalação da epífora foi de 13,2 meses, variando entre 4 e 30 meses.

Todos os pacientes apresentaram obstrução lacrimal instalada no nível do ducto nasolacrimal, logo abaixo do saco lacrimal, comprovada por sondagem, irrigação e dacriocistografia; em 64,7\% dos pacientes (11 indivíduos) a obstrução de vias lacrimais foi bilateral. Quanto ao tamanho do saco lacrimal, houve variação de grau 2 a 4, com $47 \%$ dos pacientes (8 indivíduos) apresentando saco lacrimal classificado como grau 4. Todos os pacientes foram submetidos à dacriocistorrinostomia externa com intubação do ducto nasolacrimal com silicone (Sonda de Crawford, FCl, França) com resolução total da obstrução em 82,4\% dos pacientes (14 indivíduos) e parcial em 17,6\% (três indivíduos), que apresentaram recidiva unilateral após correção bilateral da obstrução. O tempo de recidiva foi de cinco meses em dois pacientes, sendo um paciente que apresentou sangramento intraoperatório aumentado e dose cumulativa de 1200 mCi, e um

Tabela 1. Características dos pacientes e sintomas

\begin{tabular}{|c|c|c|c|c|c|c|c|c|c|c|c|}
\hline Paciente & $\begin{array}{c}\text { Idade à } \\
\text { apresentação } \\
\text { (anos) }\end{array}$ & Sexo & $\begin{array}{l}\text { Intervalo da } \\
\text { última dose de } \text { I }^{131} \\
\text { até queixa ocular } \\
\text { (meses) }\end{array}$ & $\begin{array}{l}\text { Lateralidade } \\
\text { da obstrução }\end{array}$ & $\begin{array}{c}\text { Tamanho } \\
\text { do saco } \\
\text { lacrimal }\end{array}$ & $\begin{array}{c}\text { Dose } \\
\text { cumulativa } \\
\text { de I }{ }^{131} \\
\text { (mCi) }\end{array}$ & $\begin{array}{c}\text { Complicação } \\
\text { cirúrgica }\end{array}$ & Resolução & Recidiva & $\begin{array}{c}\text { Seguimento } \\
\text { (meses) }\end{array}$ & $\begin{array}{c}\text { Sintomas } \\
\text { extraoculares* }\end{array}$ \\
\hline 1 & 43 & $\mathrm{~F}$ & 12 & Bilateral & 2 & 250 & & Sim & & 6 & \\
\hline 2 & 34 & $F$ & 24 & Unilateral & 2 & 300 & & Sim & & 5 & \\
\hline 3 & 74 & $F$ & 12 & Unilateral & 3 & 250 & & Sim & & 5 & $X S, R N, A G S$ \\
\hline 4 & 49 & $F$ & 30 & Bilateral & 2 & 250 & & Sim & & 5 & $X S, R N, P T, A G S$ \\
\hline 5 & 38 & $\mathrm{~F}$ & 14 & Bilateral & 4 & 960 & Sangramento & Sim & & 6 & \\
\hline 6 & 30 & M & 3 & Bilateral & 4 & 1200 & Sangramento & $\begin{array}{c}\text { Parcial } \\
\text { (unilateral) }\end{array}$ & $\operatorname{Sim}$ & 5 & \\
\hline 7 & 80 & M & 6 & Bilateral & 4 & 250 & & Sim & & 5 & \\
\hline 8 & 62 & $F$ & 12 & Bilateral & 2 & 650 & & $\operatorname{Sim}$ & & 2 & RN, PT, AGS \\
\hline 9 & 56 & F & 12 & Unilateral & 2 & 275 & & Sim & & 6 & $X S, R N, A G S$ \\
\hline 10 & 40 & F & 12 & Bilateral & 4 & 670 & & $\begin{array}{c}\text { Parcial } \\
\text { (unilateral) }\end{array}$ & Sim & 5 & $X S, R N, P T, A G S$ \\
\hline 11 & 44 & $F$ & 8 & Bilateral & 3 & 650 & & Sim & & 2 & $X S, R N$ \\
\hline 12 & 33 & $F$ & 12 & Unilateral & 4 & 1000 & Sangramento & $\operatorname{Sim}$ & & 2 & $X S, R N, P T, A G S$ \\
\hline 13 & 64 & F & 6 & Bilateral & 4 & 675 & & Sim & & 6 & XS, PT \\
\hline 14 & 57 & M & 24 & Bilateral & 3 & 250 & & $\begin{array}{c}\text { Parcial } \\
\text { (unilateral) }\end{array}$ & $\operatorname{Sim}$ & 48 & \\
\hline 15 & 45 & F & 18 & Unilateral & 4 & 250 & & $\operatorname{Sim}$ & & 24 & $X S, R N, P T$ \\
\hline 16 & 50 & F & 20 & Unilateral & 4 & 350 & & Sim & & 6 & \\
\hline 17 & 46 & $\mathrm{~F}$ & 4 & Bilateral & 3 & 200 & & $\mathrm{Sim}$ & & 3 & $X S, R N$ \\
\hline
\end{tabular}

*=AGS= aumento de glândula salivar; $\mathrm{RN}=$ ressecamento nasal; $\mathrm{PT}=$ parotidite; $\mathrm{XS}=$ xerostomia 
paciente com dose cumulativa de $670 \mathrm{mCi}$. O terceiro paciente apresentou recidiva 48 meses após a cirurgia, tendo recebido dose cumulativa de $250 \mathrm{mCi}$. O tempo médio de seguimento foi 6 meses, variando de 2 a 24 meses. Observou-se também dilatação de saco lacrimal (grau 4) e sangramento intraoperatório aumentado em três pacientes mais jovens, com dose cumulativa média de radioiodo igual a $1053 \mathrm{mCi}$; casos 5, 6 e 12 (respectivamente com 38, 30 e 33 anos de idade) (vide Tabela 1).

A xerostomia esteve presente em $41,1 \%$ dos pacientes (7 indivíduos), naqueles com dose cumulativa média de $616 \mathrm{mCi}$, variando de $200 \mathrm{mCi}$ a $1000 \mathrm{mCi}$. Parotidite, ressecamento nasal e aumento de outras glândulas salivares também foram relatados. Apenas um dos sete pacientes (caso 13) não relatou ressecamento nasal após a radioiodoterapia, sendo que havia recebido dose cumulativa de $675 \mathrm{mCi}$.

\section{DISCUSSÃO}

Desde a descrição de Kloos et al. em 2002 ${ }^{(10)}$, poucos artigos abordaram a obstrução de ducto nasolacrimal pós RIT, a maioria originária dos EUA e um trabalho na Alemanha. A análise da distribuição mundial do câncer de tireoide mostra que é uma das neoplasias que mais tem aumentado nos últimos anos e concentrado particularmente na América do Norte, Austrália e Europa ${ }^{(11)}$.

Consequentemente há um aumento de casos submetidos à RIT nessas regiões e a detecção de complicações relacionadas ao tratamento. No Brasil, estudo de 2011 mostra uma estimativa de incidência do câncer de tireoide em mulheres de 5,27 por 100.000 habitantes, que é comparável à incidência de alguns países europeus ${ }^{(12)}$. Espera-se assim aumento da morbidade associada ao tratamento, incluída a obstrução de ducto nasolacrimal.

O presente estudo apresenta a primeira série de pacientes brasileiros com quadro obstrutivo lacrimal associado a RIT para carcinoma de tireoide. Quanto à faixa etária e ao gênero, observamos que os pacientes se distribuem majoritariamente de acordo com o perfil epidemiológico estabelecido na literatura para carcinomas diferenciados de tireoide, qual seja, apresentação ao redor da 5a década de vida, com predomínio do sexo feminino (2), embora a observação seja de que pacientes numa faixa etária mais baixa têm sido atingidos pela afecção (casos 2, 6 e 12).

A similaridade anatômica e funcional dos sistemas lacrimal e salivar leva-nos a comparar o comprometimento desse último sistema na busca de correlações clínicas que auxiliem o diagnóstico e o tratamento relacionado ao sistema lacrimal. Sinais de acometimento de mucosa oral é descrito na literatura após a RIT, com cerca de 42,9\% de prevalência de xerostomia em alguns estudos ${ }^{(7)}$. Embora se estabeleça de maneira leve e temporária na maioria dos casos, esse sintoma pode se manifestar tardiamente e persistir anos após a administração do radiofármaco ${ }^{(7,8)}$. Alguns estudos sugerem relação diretamente proporcional entre dose cumulativa de radioiodo e intensidade da xerostomia, notadamente acima de $200 \mathrm{mCi}{ }^{(8)}$. Na casuística apresentada, a incidência de xerostomia $(41,1 \%)$ e a dose cumulativa de radioiodo (200 a $1000 \mathrm{mCi}$, média de $616 \mathrm{mCi}$ ) foram concordantes com os achados de outros autores ${ }^{(7,8)}$.

Já a sialoadenite compromete mais frequentemente as glândulas parótidas, de forma bilateral. Solans e colaboradores relatam incidência de 22,8\% em série de 79 pacientes, com maior comprometimento parotídeo bilateral ${ }^{(8)}$. As glândulas salivares apresentam grande habilidade de concentração de radioiodo, fato que induziria alterações na composição salivar, como aumento da amilase e ativação de calicreínas, e causaria processo obstrutivo, redundando em redução da secreção(13-15). Dentre os pacientes que apresentaram sialoadenite, 85,7\% (6 indivíduos) desenvolveram parotidite, e um paciente apresentou envolvimento apenas de outra glândula salivar.

A presença de sintomas oculares é descrita por alguns autores. Solans e colaboradores ${ }^{(8)}$ descrevem a xeroftalmia como complicação da radioiodo nos casos apresentados, sempre associada à xerostomia.
Embora a associação entre a dose cumulativa de radioiodo e a disfunção de glândulas salivares esteja bem documentada, o mesmo não se pode categoricamente afirmar em relação à dose do radiofármaco e sintomas oculares.

Recentemente, Kloos et al. ${ }^{(10)}$ relataram epífora em 10 pacientes, dentre uma casuística de 423 indivíduos submetidos a RIT. Dentre eles, todos apresentaram evidência de acometimento do sistema de drenagem lacrimal, sendo que $50 \%$ dos casos (5 pacientes) apresentaram epífora bilateral, e 90\% (9 pacientes) evidenciaram algum comprometimento do ducto nasolacrimal. A dose cumulativa média de radioiodo foi de $467+79 \mathrm{mCi}$, o tempo médio de surgimento de sintomas de 6,5 + 1,4 meses após a última dose de radioiodo e o intervalo médio entre o início dos sintomas de obstrução lacrimal e o diagnóstico correto de $18+5$ meses. Nenhum paciente tratado com dose de $150 \mathrm{mCi}$ ou menor apresentou obstrução de ducto nasolacrimal. Em estudo do mesmo grupo de pesquisadores, Burns et al. ${ }^{(16)}$ avaliaram, dentre 563 pacientes submetidos a RIT, 26 indíviduos com queixa de epífora, através de sondagem e irrigação de vias lacrimais. Desses pacientes, 10 pacientes eram provenientes do estudo citado anteriormente e 6 pacientes eram novos casos, com $80 \%$ desses (4 indivíduos) apresentando algum comprometimento de ducto nasolacrimal. Outros locais de obstrução foram canalículo comum e canalículo distal. A dose cumulativa média foi de $525 \mathrm{mCi}$ (200 a 951 mCi), e o sintoma de epífora surgiu após 8,5 meses do tratamento (1 a 15 meses). O tempo médio de início dos sintomas até o diagnóstico correto foi de 13 meses, fato que evidencia, juntamente com o estudo de Kloos e colaboradores, a demora do diagnóstico pelo desconhecimento da associação entre o uso da RIT e o surgimento de distúrbios da drenagem lacrimal.

Em nossa casuística, a dose cumulativa média dos 17 pacientes foi semelhante $(571 \mathrm{mCi})$, com dose mínima de $200 \mathrm{mCi}$ (acima portanto da dose de $150 \mathrm{mCi}$, como cita Burns e colaboradores), constituindo amostra dentro do risco de obstrução da drenagem lacrimal ${ }^{(13)}$. A obstrução do ducto nasolacrimal ocorreu em todos os 17 casos e comprometimento bilateral em $64,7 \%$ (11 pacientes). O intervalo médio entre a última dose de RIT e o surgimento de epífora também foi semelhante (13,2 meses) aos estudos de Kloos e colaboradores.

Embora seja um dos principais tratamentos do hipertireoidismo da doença de Graves, a RIT não parece estar associada à obstrução de vias lacrimais nesses casos, provavelmente pela dose menor do radiofármaco, normalmente de 10 a 15 mCi ${ }^{(16)}$, como observou Shepler em seu estudo ${ }^{(17)}$.

O dilatação acentuada do saco lacrimal em 47\% dos pacientes (8 indivíduos) é achado condizente com o diagnóstico de obstrução baixa e curso crônico do quadro. As dimensões do saco lacrimal não são relatadas em outros estudos sobre o tema. Quanto à recidiva da obstrução, Kloos et al. ${ }^{(10)}$ não explicitam o tempo de seguimento, porém dos 10 pacientes sintomáticos, 3 foram submetidos a dacriocistorrinostomia externa, com resolução total do quadro em 2 pacientes e parcial em 1 paciente, que apresentava obstrução bilateral; em 4 pacientes foi optado pela observação e em 3 pacientes foram realizadas dacrioplastia por balão e intubação com silicone, sendo que não apresentaram melhora ou apresentaram melhora parcial, necessitando de novos procedimentos.

A obstrução do ducto nasolacrimal parece estar relacionada à presença do cotransportador sódio-iodo na mucosa do ducto nasolacrimal(18), justificando a captação do radioiodo e consequentes inflamação e fibrose, culminando no processo obstrutivo e no quadro clínico de epífora e dacriocistite.

Nos estudos que abordam o tema, os pacientes foram avaliados apenas nos casos em que apresentaram queixa, da mesma forma pela qual foram encaminhados ao nosso atendimento. Talvez o comprometimento da via lacrimal esteja subdiagnosticado ou não-diagnosticado em grande parte dos casos. Não encontramos trabalhos prospectivos que visem avaliar a real incidência da obstrução de vias lacrimais, bem como seu tempo de instalação. É possível que a 
obstrução se estabeleça precocemente e não seja relatada, eventualmente por ocorrer de maneira concomitante à xeroftalmia e assim não se manifestar precocemente.

\section{CONCLUSÃO}

A radioiodoterapia se apresenta em estudos recentes como novo fator etiológico da obstrução adquirida de vias lacrimais. Entretanto, estudos prospectivos avaliando o impacto da RIT na produção e drenagem lacrimais são necessários para melhor conhecimento da repercussão do tratamento sobre o sistema lacrimal, de maneira que possamos melhor compreender sua etiopatogenia, as diversas variáveis envolvidas e estudar meios de intervenção que possibilitem minimizar ou impedir a instalação definitiva da obstrução lacrimal.

\section{REFERÊNCIAS}

1. Schmid KE, Kornek GV, Scheithauer W, Binder S. Update on ocular complications of systemic cancer chemotherapy. Surv Ophthalmol. 2006;51(1):19-40.

2. Mazzaferri EL, Kloos RT. Clinical review 128: Current approaches to primary therapy for papillary and follicular thyroid cancer. J Clin Endocrinol Metab. 2001;86(4):1447-63.

3. Davies L, Welch HG. Increasing incidence of thyroid cancer in the United States, 19732002. JAMA. 2006;295(18):2164-7.

4. Samaan NA, Schultz PN, Hickey RC, Goepfert H, Haynie TP, Johnston DA, Ordonez NG. The results of various modalities of treatment of well differentiated thyroid carcinomas: a retrospective review of 1599 patients. J Clin Endocrinol Metab. 1992;75(3):714-20.

5. Bohuslavizki KH, Brenner W, Lassmann S, Tinnemeyer S, Tönshoff G, Sippel C, et al. Quantitative salivary gland scintigraphy in the diagnosis of parenchymal damage after treatment with radioiodine. Nucl Med Commun. 1996;17(8):681-6.

6. Markitziu A, Lustmann J, Uzieli B, Krausz Y, Chisin R. Salivary and lacrimal gland in- volvement in a patient who had undergone a thyroidectomy and was treated with radioiodine for thyroid cancer. Oral Surg Oral Med Oral Pathol. 1993;75(3):318-22.

7. Alexander C, Bader JB, Schaefer A, Finke C, Kirsch CM. Intermediate and long-term side effects of high-dose radioiodine therapy for thyroid carcinoma. J Nucl Med. 1998; 39(9):1551-4.

8. Solans R, Bosch JA, Galofré P, Porta F, Roselló J, Selva-O'Callagan A, Vilardell M. Salivary and lacrimal gland dysfunction (sicca syndrome) after radioiodine therapy. J Nucl Med. 2001:42(5):738-43.

9. Fard-Esfahani A, Mirshekarpour H, Fallahi B,Eftekhari M, Saghari M, Beiki D, et al. The effect of high-dose radioiodine treatment on lacrimal gland function in patients with differentiated thyroid carcinoma. Clin Nucl Med. 2007;32(9):696-9.

10. Kloos RT, Duvuuri V, Jhiang SM, Cahill KV, Foster JA, Burns JA. Nasolacrimal drainage system obstruction from radioactive iodine therapy for thyroid carcinoma. J Clin Endocrinol Metab. 2002;87(12):5817-20.

11. Ferlay J, Shin JR, Bray F, Forman D, Mathers C, Parkin DM. GLOBOCAN 2008 v1.2, Cance Incidence and Mortality Worldwide: IARC Cancer Base No.10 [Internet]. Lyon, France: International Agency for Research on Cancer, 2010. Available from: http://globocan.iarc.fr.

12. Brito AS, Coeli CM, Barbosa FS, Caetano R, Santos MO, Vaisman M. Estimates of thyroid cancer incidence in Brazil: an approach using polynomial models. Cad Saúde Pública. 2011:27(7):1441-4

13. Allweiss P, Braunstein GD, Katz A, Waxman A. Sialadenitis following $1-131$ therapy for thyroid carcinoma: concise communication. J Nucl Med. 1984;25(7):755-8.

14. Levenson D, Gulec S, Sonenberg M, Lai E, Goldsmith SJ, Larson SM. Peripheral facial nerve palsy after high-dose radioiodine therapy in patients with papillary thyroid carcinoma. Ann Intern Med. 1994;120(7):576-8.

15. DiRusso G, Kern KA. Comparative analysis of complications from 1-131 radioablation for well-differentiated thyroid cancer. Surgery. 1994;116(6):1024-30.

16. Burns JA, Morgenstern KE, Cahill KV, Foster JA, Jhiang SM, Kloos RT. Nasolacrimal obstruction secondary to I(131) therapy. Ophthal Plast Reconstr Surg. 2004:20(2):126-9.

17. Shepler TR, Sherman SI, Faustina MM, Busaidy NL, Ahmadi MA, Esmaeli B. Nasolacrimal duct obstruction associated with radioactive iodine therapy for thyroid carcinoma. Ophthal Plast Reconstr Surg. 2003;19(6):479-81.

18. Leder $\mathrm{O}$. The significance of extrathyroidal radioactive iodine accumulation and secretion in clinical pathology. Histochemistry. 1982;74(4):585-8.

\title{
XVII Congresso Internacional da Sociedade Brasileira de Oftalmologia
}

\author{
28 a 30 de junho de 2012 \\ Hotel Royal Tulip - Rio de Janeiro (RJ)
}

Informações:

INTEREVENT - Tel.: (21) 3326-3320 - Fax: (21) 2437-1483

E-mail: oftalmologia@interevent.com.br

Site: www.sboportal.org.br 